\title{
Improving the Management of an Advanced Extrauterine Pregnancy Using Pelvic Arteriography in a Hybrid Operating Suite
}

\author{
Michael P. Smrtka, M.D. ${ }^{1}$ Ravindu Gunatilake, M.D. ${ }^{1}$ \\ Haywood L. Brown, M.D. 1 \\ ${ }^{1}$ Division of Maternal-Fetal Medicine, Department of Obstetrics and \\ Gynecology, Duke University Medical Center, Durham, North \\ Carolina \\ 2 Division of Interventional Radiology, Duke University Medical Center, \\ Durham, North Carolina
}

\author{
Michael J. Miller, M.D. ${ }^{2}$
}

R. Phillips Heine, M.D. ${ }^{1}$

\begin{abstract}
Address for correspondence and reprint requests Michael P. Smrtka, M.D., Division of Maternal-Fetal Medicine, Department of Obstetrics and Gynecology, Duke University Medical Center, DUMC Box 3967, Durham, NC 27710 (e-mail: michael.smrtka@duke.edu).
\end{abstract}

Am J Perinatol Rep 2012;2:63-66.

\begin{abstract}
Keywords

- advanced extrauterine pregnancy

- abdominal pregnancy

- pelvic arteriography

Advanced extrauterine pregnancy is an extremely rare, life-threatening pregnancy complication. Management of these pregnancies presents significant challenges, especially when they have progressed to an advanced stage of fetal viability. With high rates of maternal and fetal mortality associated with this complication, delivery or pregnancy interruption should be expedited following diagnosis. Localization of the placenta and its blood supply is critical to preoperative planning. Hybrid operating suites that can accommodate a multidisciplinary team of subspecialists may improve the chance of a successful outcome with this rare complication.
\end{abstract}

Advanced extrauterine pregnancy is an extremely rare, lifethreatening pregnancy complication, occurring in an estimated 1 per 10,000 births. ${ }^{1,2}$ Asymptomatic cases may progress undiagnosed to an advanced stage, even to the point of fetal viability. Given the potential for abnormal placentation and catastrophic bleeding at delivery or pregnancy interruption, localization of the placenta and its blood supply is critical to preoperative planning. Hybrid operating suites (HOSs) combine the equipment and resources of a vascular interventional radiology suite with a traditional operating room that can accommodate a multidisciplinary team of subspecialists and enhances imaging and resuscitative capabilities. The ability to rapidly adapt the surgical approach and incorporate multiple surgical specialists or interventionalists without transporting a potentially unstable patient highlights the advantages of an HOS in the setting of advanced extrauterine pregnancy, thereby improving the chance of a successful outcome with this rare complication.

\section{Case Report}

We present the case of a 21-year-old gravida 3, para 2-0-2-2 admitted at 23 weeks and 6 days of gestation from a referral hospital for management of an advanced extrauterine pregnancy. Three weeks prior to admission, the patient presented to her local health department to establish prenatal care at 15 weeks' gestation based on an uncertain last menstrual period. Two days prior to admission, a routine anatomy ultrasound raised concern for an intra-abdominal pregnancy. The patient denied any pelvic pain and was completely asymptomatic up to that point in her pregnancy. She was transferred immediately to a regional medical center for additional imaging and consultation with a perinatologist. During that time, she received a course of antenatal corticosteroids. Ultrasound demonstrated a live left-sided abdominal pregnancy at 23 weeks and 5 days of gestation and anhydramnios with an estimated fetal weight of $443 \mathrm{~g}$ (less received

March 3, 2012

accepted after revision

April 1, 2012

published online

July 16, 2012
Copyright $\odot 2012$ by Thieme Medical Publishers, Inc., 333 Seventh Avenue, New York, NY 10001, USA. Tel: +1(212) 584-4662.
DOI http://dx.doi.org/ 10.1055/s-0032-1322507. ISSN 2157-6998. 
than fifth percentile overall; head circumference measured 25 weeks 5 days). Magnetic resonance imaging (MRI) confirmed the diagnosis of an extrauterine pregnancy and suggested placentation over the maternal lumbosacral spine.

At this point, the patient was transferred to our institution for further management and likely delivery. Following admission, the diagnosis of a live, advanced extrauterine pregnancy with anhydramnios was confirmed sonographically. The patient and her family were counseled that these findings conveyed an extremely poor fetal prognosis and that continuing the pregnancy placed the mother at risk for severe morbidities and significant risk of mortality. In agreement with our recommendations, the patient consented to delivery and requested attempted salvage of the fetus.

We enlisted a multidisciplinary team of specialists in vascular-interventional radiology, vascular surgery, obstetric anesthesiology, gynecologic oncology, colorectal surgery, and neonatology to aid in planning and coordination of the procedure. The delivery and surgery were scheduled in one of our HOSs to allow for perioperative arteriography and potential embolization by an interventional radiologist. A contrasted magnetic resonance arteriogram was obtained preoperatively to further delineate the vascular supply to the placenta. The bilobed placenta appeared to have two distinct components with respective blood supplies arising from the right common iliac and internal iliac arteries (-Figs. 1 and 2). Multiple units of blood products were matched for our procedure. Once in the HOS, the patient underwent epidural placement and general anesthesia. The right common femoral artery was accessed and a 5-French angiography catheter was advanced to the abdominal aorta by our interventional radiologist. Pelvic arteriography was performed with Isovue ${ }^{\circledR}-300$ contrast (iopamidol, Bracco Diagnostics, Princeton, NJ) demonstrating the arterial supply to the placental components from the left ovarian and left

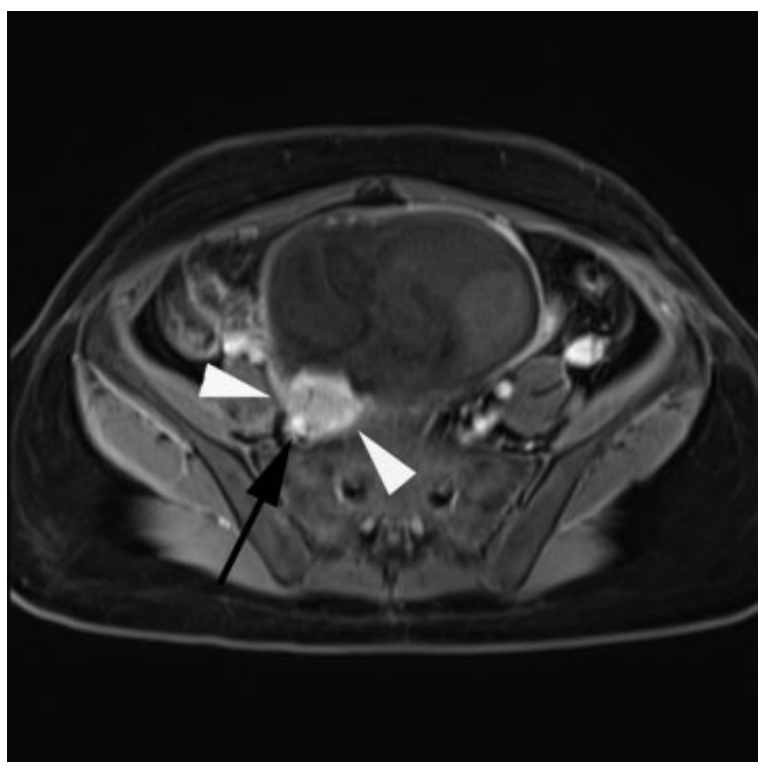

Figure 1 Axial postgadolinium image demonstrates enhancing placental tissue (white arrowheads) adjacent to the right common femoral artery (black arrow) with loss of the normal fat tissue plane.

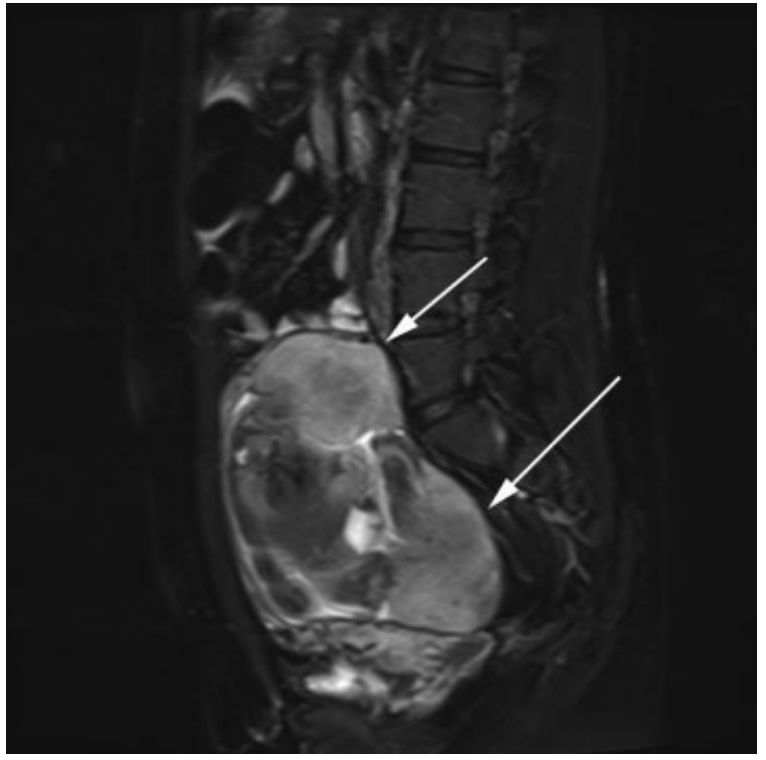

Figure 2 Sagittal postgadolinium image demonstrates the bilobed placenta (arrows) enhancing with effacement against the spine.

uterine arteries primarily (-Fig. 3). There appeared to be minimal vascular recruitment from the right-sided lumbar vessel coming off the most inferior aspect of the abdominal aorta. The angiography catheter was removed and the groin access maintained for possible embolization if needed.

We proceeded to exploratory laparotomy and identified a large, fibrotic gestational sac extruding from a massively dilated left tube and fimbria as well as a nonpregnant uterus (-Fig. 4). The gestational sac was incised well away from any perceived placental attachments, and within the sac, a flaccid amniotic sac containing the fetus and minimal amniotic fluid was visualized. The amniotic sac was incised and the fetus

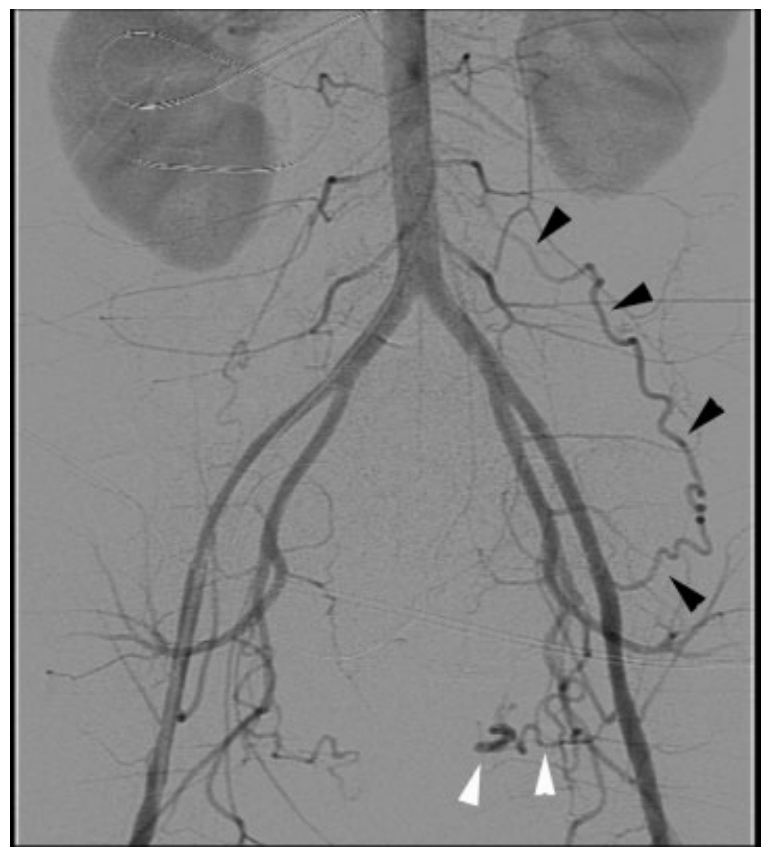

Figure 3 Image from arteriogram prior to delivery demonstrates arterial supply to the placenta from an enlarged left ovarian artery (black arrowheads) and the left uterine artery (white arrowheads). 


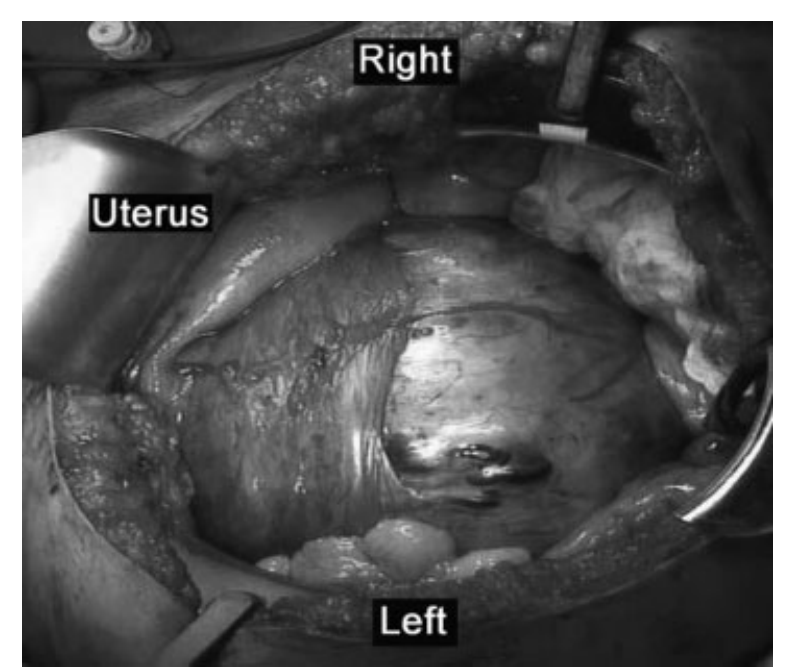

Figure 4 Intraoperative findings of nonpregnant uterus and large, fibrotic gestational sac extruding from massively dilated left tube and fimbria.

delivered gently and handed to the awaiting pediatric resuscitation team. The live-born female infant was intubated, assigned Apgar scores of 5 at 1 minute and 7 at 5 minutes, and weighed $580 \mathrm{~g}$.

Preoperatively, the patient was counseled that we would most likely leave the placenta in situ given the suspected vascular involvements and this approach has been supported in the available literature as a means of preventing severe hemorrhage. ${ }^{4}$ However, after delivery of the fetus, further inspection of the gestational sac demonstrated attachments to the rectosigmoid colon and left ovary. As the pregnancy sac appeared relatively encapsulated with the vascular supply clearly delineated by the arteriogram, it was felt that a left salpingo-oophorectomy could be accomplished safely with minimal blood loss and operative risk. During the dissection and resection, the patient sustained a 1-cm enterotomy, which was repaired after removal of the remaining pregnancy tissue. Because all pregnancy tissue was removed and hemostasis was obtained with minimal blood loss of $600 \mathrm{~mL}$, postoperative arteriography with embolization was not required. The patient was discharged home on postoperative day 7 , after having regained normal bowel function and meeting all early milestones for discharge.

Despite initial concerns for pulmonary hypoplasia in the setting of anhydramnios, the neonate experienced a relatively uncomplicated neonatal course. The infant was extubated on day 3 of life and advanced to enteral feeds. A normal cranial ultrasound on day 7 of life and again at 5 weeks of life suggested the absence of intraventricular hemorrhage. The neonate remained in the intensive and special care nurseries for the first 5 months of life and weighed $3135 \mathrm{~g}$ at the time of discharge.

\section{Discussion}

Similar to other forms of invasive placentation such as placenta percreta, predelivery diagnosis of advanced extrauterine pregnancy is critical to minimizing maternal-fetal morbidity and mortality. Identifying the exact origin of placental vascular supply can facilitate optimal surgical management. Though previous case reports of advanced extrauterine pregnancy are subject to publication bias with unreported poor outcomes, we can certainly infer that placental attachment to vital structures such as iliac vessels or bowel carry a higher level of potential morbidity in comparison to a pregnancy mostly encapsulated within a fallopian tube. The exact site of placentation can vary to include tubal, peritoneal, omental, and bowel attachment sites. Rather than "abdominal pregnancy," the terminology of advanced extrauterine pregnancy appears to better characterize this rare pregnancy complication.

In our case, ultrasound and MRI were important diagnostic modalities in guiding the decision and timing of delivery. However, the favorable outcome underscores the usefulness of arteriography within an HOS in identifying the vascular source of the placenta and guiding our surgical approach. Based on the preoperative pelvic MRI, the placental attachment site was initially felt to be the sacral promontory with vascular supply from the right iliac artery. In contrast, predelivery arteriography revealed the left ovarian artery to be the primary placental vascular supply for the pregnancy. Immediately following laparotomy, we confirmed the extrauterine pregnancy to be mostly encapsulated and confined within the left fallopian tube with partial placental extrusion and attachment to the rectosigmoid colon. Knowledge of the primary vascular source of the placenta guided our dissection of adhesions, incision through the pregnancy sac, and ultimately the decision to remove the placenta in its entirety and perform a left salpingo-oophorectomy following ligation of the left ovarian artery.

Given the wide variation in placental attachment sites among reported cases of advanced extrauterine pregnancy, it is not surprising that the decision for placental removal is controversial. ${ }^{3-6}$ Nevertheless, this case demonstrates that an individualized approach with complete removal of the placenta guided by pelvic arteriography can be reasonably accomplished in specialized perinatal centers. ${ }^{6}$ We were prepared for massive hemorrhage though blood loss was minimal in this case. Ligation of the primary vascular supply following delivery of the infant minimized bleeding during the remainder of the surgery. The femoral catheter sheaths were left in place following arteriography for the remainder of the delivery and surgical excision. The immediate availability of vascular access provides the option to rapidly insert vaso-occlusive "balloon" catheters should massive hemorrhage occur.

Our institution recently developed an HOS with the capability to accommodate multiple surgical disciplines including interventional radiology, vascular surgery, surgical oncology, and cardiothoracic surgery. The HOS incorporates ample space and equipment for handling resuscitation associated with critical illness such as vascular trauma while seamlessly incorporating interventional procedures. For cases with the potential for massive obstetric hemorrhage, the HOS offers the capability for interventionists to place and inflate pelvic balloon catheters or perform embolization to control 
hemorrhage without moving an already unstable patient to a traditional interventional radiology suite. Most importantly, it offers the flexibility to rapidly modify a surgical approach and incorporate multiple surgical specialties with minimal preparation. When available, obstetric cases of placenta percreta and advanced extrauterine pregnancy are ideally suited for management in these hybrid suites.

Advanced extrauterine pregnancy presents several diagnostic and management challenges. Given the potential for poor fetal growth and congenital abnormalities associated with abnormal placentation, a detailed perinatal ultrasound is recommended upon diagnosis to verify pregnancy dating as this information is critical for delivery planning. When possible, we recommend that delivery occur in a specialized perinatal center with immediate availability of interventional and surgical consultants. When available, an HOS is the optimal setting for delivery given the need for flexibility in surgical approach and potential for severe maternal hemorrhage even with the best intentions of leaving the placenta in situ. With such a rare complication, it is challenging to provide definitive recommendations regarding timing of delivery. The high risk of maternal mortality argues for immediate delivery at the time of diagnosis. Alternatively, if expectant management is selected following thorough informed consent, it should not exceed a gestational age associated with fetal viability. Risk with advanced extrauter- ine pregnancy remains high. However, optimal preparation, resources, and early diagnosis can result in a favorable outcome, as was the case for the mother and neonate presented in this report.

\section{References}

1 Atrash HK, Friede A, Hogue CJ. Abdominal pregnancy in the United States: frequency and maternal mortality. Obstet Gynecol 1987; 69(3 Pt 1):333-337

2 Hall JM, Manning N, Moore NR, Tingey WR, Chamberlain P. Antenatal diagnosis of a late abdominal pregnancy using ultrasound and magnetic resonance imaging: a case report of successful outcome. Ultrasound Obstet Gynecol 1996;7:289-292

3 Nkusu Nunyalulendho D, Einterz EM. Advanced abdominal pregnancy: case report and review of 163 cases reported since 1946. Rural Remote Health 2008;8:1087

4 Oneko O, Petru E, Masenga G, Ulrich D, Obure J, Zeck W. Management of the placenta in advanced abdominal pregnancies at an East African tertiary referral center. J Womens Health (Larchmt) 2010;19:1369-1375

5 Oki T, Baba Y, Yoshinaga M, Douchi T. Super-selective arterial embolization for uncontrolled bleeding in abdominal pregnancy. Obstet Gynecol 2008;112(2 Pt 2):427-429

6 Worley KC, Hnat MD, Cunningham FG. Advanced extrauterine pregnancy: diagnostic and therapeutic challenges. Am J Obstet Gynecol 2008;198:297, e1-e7 